ARTICLE

https://doi.org/10.1038/s41467-019-08542-1

\title{
Designing chemical analogs to PbTe with intrinsic high band degeneracy and low lattice thermal conductivity
}

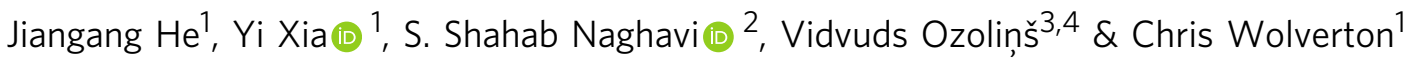

High-efficiency thermoelectric materials require simultaneously high power factors and low thermal conductivities. Aligning band extrema to achieve high band degeneracy, as realized in $\mathrm{PbTe}$, is one of the most efficient approaches to enhance power factor. However, this approach usually relies on band structure engineering, e.g., via chemical doping or strain. By employing first-principles methods with explicit computation of phonon and carrier lifetimes, here we show two full-Heusler compounds $\mathrm{Li}_{2} \mathrm{TIBi}$ and $\mathrm{Li}_{2} \mathrm{InBi}$ have exceptionally high power factors and low lattice thermal conductivities at room temperature. The expanded rock-salt sublattice of these compounds shifts the valence band maximum to the middle of the $\Sigma$ line, increasing the band degeneracy by a factor of three. Meanwhile, resonant bonding in the $\mathrm{PbTe}$-like sublattice and soft $\mathrm{TI}-\mathrm{Bi}$ ( $\mathrm{In}-\mathrm{Bi}$ ) bonding interaction is responsible for intrinsic low lattice thermal conductivities. Our results present an alternative strategy of designing high performance thermoelectric materials.

\footnotetext{
${ }^{1}$ Department of Materials Science and Engineering, Northwestern University, Evanston, IL 60208, USA. ${ }^{2}$ Department of Physical and Computational Chemistry, Shahid Beheshti University, G.C., Evin, Tehran 1983969411, Iran. ${ }^{3}$ Department of Applied Physics, Yale University, New Haven, CT 06520, USA. ${ }^{4}$ Yale Energy Sciences Institute, West Haven, CT 06516, USA. These authors contributed equally: Jiangang He, Yi Xia. Correspondence and requests for materials should be addressed to C.W. (email: c-wolverton@northwestern.edu)
} 
T hermoelectric (TE) materials have important applications in energy harvesting, thermoelectric coolers, and thermal detectors as they can directly convert heat into electricity and vise versa. Highly efficient TE materials are required for practical applications and are characterized by the figure of merit $z T=\left(S^{2} \sigma T\right) /\left(\kappa_{\mathrm{L}}+\kappa_{\mathrm{e}}\right)$, where $S, \sigma, \kappa_{\mathrm{e}}, \kappa_{\mathrm{L}}$, and $T$ are the Seebeck coefficient, electrical conductivity, electronic thermal conductivity, lattice thermal conductivity, and temperature, respectively. In order to maximize $z T$, both electronic transport properties and lattice thermal conductivity have to be optimized carefully. Many strategies have been successfully used to suppress $\kappa_{\mathrm{L}}{ }^{1}$. However, there are fewer approaches that can effectively improve the electronic properties, i.e., the power factor $\left(\mathrm{PF}=S^{2} \sigma\right)$ $2-4$. One effective route is to increase the band degeneracy $\left(N_{\mathrm{v}}\right)$ and decrease the inertial effective mass $\left(m_{\mathrm{I}}^{*}\right)$ simultaneously since the figure of merit $z T$ of a material is proportional to $\frac{N_{\mathrm{v}} 2,5}{m_{\mathrm{T}}^{*}}$. Although a high density of states (DOS) effective mass $\left(m_{\mathrm{d}}^{*}\right.$ $=N_{\mathrm{v}}^{2 / 3} m_{\mathrm{b}}^{*}$ ) is preferred for generating a high $S^{6,7}$, the band effective mass $m_{\mathrm{b}}^{*}$ is also concomitantly high in a material with low $N_{\mathrm{v}}$, leading to a low electrical conductivity as $\sigma \propto \frac{\tau}{m_{\mathrm{b}}^{*}}(\tau$ is the carrier lifetime) $)^{5}$.

A high value of $N_{\mathrm{v}}$ can be achieved either from a high valley multiplicity (the number of the carrier pockets of a band in the Brillouin zone) or a high orbital degeneracy (the number of bands with the same energy). Take the well studied TE material $\mathrm{PbTe}$ (rock-salt lattice, space group $F m \overline{3} m$ ) as an example, once the second maximum of the valence band (the middle of the $\Sigma$ line, multiplicity is 12) is converged with the valence band maximum (VBM) (at the L point, multiplicity is 4) by alloying an appropriate amount $\mathrm{PbSe}$, a significant enhancement of $z T$ from 0.8 to 1.8 can be reached ${ }^{8}$. In practice, many materials have very limited dopability or the energy band can not be properly converged. Therefore, TE materials with intrinsically high band degeneracy are highly desired. Unfortunately, most intrinsic semiconductors have very low valley multiplicity. A high valley multiplicity usually only appears in cubic crystal systems where the VBM or conduction band minimum (CBM) is located at a low symmetry point of the first Brillouin zone, such as the $\Sigma$ line of the rock-salt structure $^{2}$. In addition to alloying, the band convergence could, in principle, be achieved through strain engineering. The lattice constant plays an important role on the alignment of $\Sigma$ and L in $\mathrm{PbTe}^{8,9}$. However, a completely alignment requires an extremely large strain, which is not reachable in practice. Therefore, an alternative material design strategy is desired.

Semiconducting half-Heusler (HH) (chemical formula XYZ; space group $F \overline{4} 3 m$ ) compounds have been widely studied as TE materials due to their high power factors ${ }^{10-12}$ and excellent dopability ${ }^{13,14}$. Since semiconducting full-Heusler (FH) (chemical formula $X_{2} Y Z$; space group $F m \overline{3} m$ ) compounds are very rare, the study of FH TE is highly limited ${ }^{15}$. Owing to the structural similarity between $\mathrm{HHs}$ and $\mathrm{FHs}^{16}$, semiconducting $\mathrm{FH}$ compounds are expected to have good TE performance as well. The FH structure is a face centered cubic crystal structure with the interpenetration of $X_{2}$ cubic and $Y Z$ rock-salt sublattices. The embedded cubic sublattice extends the bond length between $Y$ and $Z$ atoms of the rock-salt sublattice. Therefore, $\mathrm{FH}$ structure is an ideal candidate for realizing expanded $\mathrm{PbTe}$.

In this work, one stable $\left(\mathrm{Li}_{2} \mathrm{TlBi}\right)$ and one metastable $\left(\mathrm{Li}_{2} \mathrm{InBi}\right)$ $\mathrm{FH}$ compounds with $\mathrm{PbTe}$-like electronic structure, are discovered by combining a TE material design strategy and high throughput ab initio thermodynamic screening ${ }^{15,17}$, see Supplementary Note 1 for details. The crystal structure of $\mathrm{FH} \mathrm{Li} \mathrm{L}_{2} \mathrm{TlBi}$ $\left(\mathrm{Li}_{2} \mathrm{InBi}\right)$ is the interpenetration of $\mathrm{Li}_{2}$ cubic and TlBi (In-Bi) rock-salt sublattices. The electronic structure of $\left[\mathrm{Li}^{+}\right]_{2}\left[\mathrm{Tl}^{+} \mathrm{Bi}^{3-}\right]$ $\left(\left[\mathrm{Li}^{+}\right]_{2}\left[\mathrm{In}^{+} \mathrm{Bi}^{3-}\right]\right)$ is isoelectronic with $\mathrm{PbTe}\left(\mathrm{Pb}^{2+} \mathrm{Te}^{2-}\right)$ since the electrons donated by two $\mathrm{Li}$ atoms are delocalized in the whole system. However, the bond length of $\mathrm{Tl}-\mathrm{Bi}$ (In-Bi) is considerably extended in the FH lattice. Consequently, both the VBM and CBM of these two compounds are located in the middle of the $\Sigma$ line, with band degeneracy of $N_{\mathrm{v}}=12$ in the intrinsic compounds due to their large lattice constants $(\sim 7.15 \AA)$. Our transport calculations, explicitly including phonon-phonon and electron-phonon interactions, show that these two compounds have low $\kappa_{\mathrm{L}}$ and high $\mathrm{PF}$ at room temperature. Benefiting from their low $\kappa_{\mathrm{L}}$ and high $\mathrm{PF}, \mathrm{Li}_{2} \mathrm{InBi}$ and $\mathrm{Li}_{2} \mathrm{TlBi}$ are therefore identified as promising room-temperature TE materials with ideal $z T$ values of 1.5 and 2.0 at $300 \mathrm{~K}$, respectively.

\section{Results}

Stability. Our density functional theory (DFT) calculations show that $\mathrm{FH} \mathrm{Li}_{2} \mathrm{TlBi}$ is on the $T=0 \mathrm{~K}$ convex hull, which means it is thermodynamically stable at zero Kelvin, and $\mathrm{Li}_{2} \mathrm{InBi}$ is just 4 $\mathrm{meV} /$ atom above the convex hull, which indicates it is thermodynamically weakly unstable (metastable). The convex hull distance (stability) is the formation energy difference between the target compound and its competing phases included in the Open Quantum Material Database (OQMD) ${ }^{18}$, which contains over 650,000 DFT calculations consisting of experimentally observed compounds from the international crystal structure database (ICSD $)^{19,20}$ and hypothetical compounds with prototype structures. We also performed a ground state crystal structure search by using 21 distinct $X_{2} Y Z$ prototype structures (see Supplementary Table 1) and the particle swarm optimization method as implemented in the CALYPSO $\operatorname{code}^{21,22}$, and we find the $\mathrm{FH}$ structure (see Fig. 1) is the lowest energy structure for both $\mathrm{Li}_{2} \mathrm{InBi}$ and $\mathrm{Li}_{2} \mathrm{TlBi}$. Phonon calculations show that both $\mathrm{Li}_{2} \mathrm{TlBi}$ and $\mathrm{Li}_{2} \mathrm{InBi}$ are dynamically stable at $T=0 \mathrm{~K}$. Free energy differences $(\Delta G=\Delta H-T \Delta S$, where $\Delta H$ and $\Delta S$ are formation energy and formation entropy, respectively, see Supplementary Table 2 for details.) between FH phases and their competing phases in the corresponding ternary phase space show that $\mathrm{Li}_{2} \mathrm{TlBi}$ is thermodynamically stable and $\mathrm{Li}_{2} \mathrm{InBi}$ is slight unstable ( $\sim 4 \mathrm{meV} /$ atom above the convex hull) up to $500 \mathrm{~K}$, see Supplementary Fig. 1 . We count $\mathrm{Li}_{2} \mathrm{InBi}$ as synthesizable compound since such small convex hull distance is well within the threshold of Heusler compounds ${ }^{23,24}$.
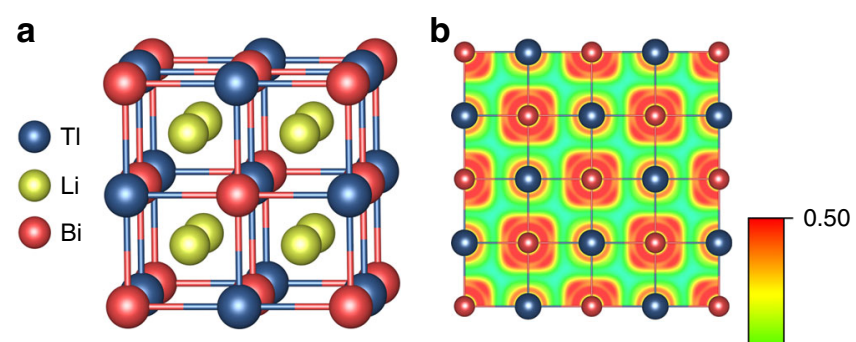

c
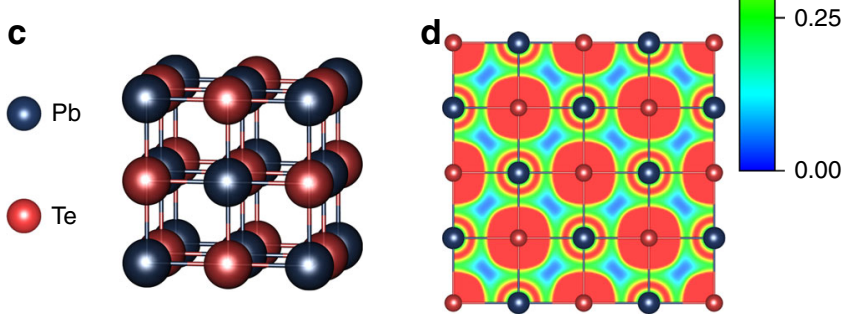

Fig. 1 Crystal and electronic structures. a Crystal structure of full Heusler $\mathrm{Li}_{2} \mathrm{TIBi}$. b Electron localization function (ELF) of $\mathrm{Li}_{2} \mathrm{TIBi}$ in (001) plane. c Crystal structure of PbTe. d ELF of PbTe in (001) plane 
Electronic structure. The main features of the $\mathrm{Li}_{2} Y \mathrm{Bi}(Y=\mathrm{In}$ and $\mathrm{Tl})$ band structure are determined by $\left[\mathrm{Y}^{+} \mathrm{Bi}^{3-}\right]^{2-}$, which is isoelectronic with $\mathrm{Pb}^{2+} \mathrm{Te}^{2-}$ even though $\mathrm{In} / \mathrm{Tl}(\mathrm{Bi})$ is cubiccoordinated with eight $\mathrm{Li}$ atoms as the nearest neighbors and
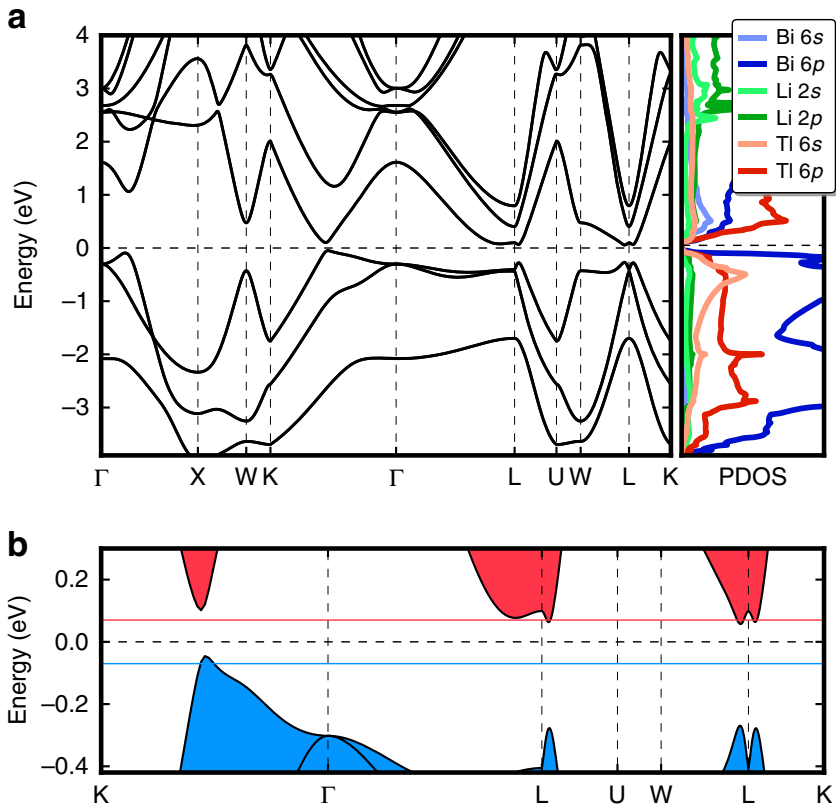

C

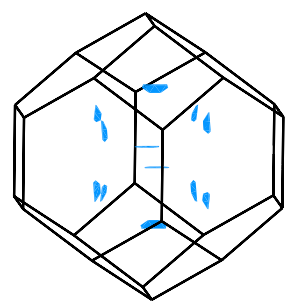

d

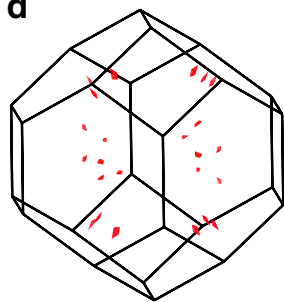

Fig. 2 Electronic structure of $\mathrm{Li}_{2} \mathrm{~T} \mid \mathrm{Bi}$. a Band structure (left) and density of states (right) of $\mathrm{Li}_{2} \mathrm{TIBi}$. $\mathbf{b}$ Expanded view of the band structure around the Fermi level. c Fermi surface of hole doped $\mathrm{Li}_{2} \mathrm{TIBi}$. d Fermi surface of electron doped $\mathrm{Li}_{2} \mathrm{TIBi}$. Note the size of Fermi pockets in electron doped $\mathrm{Li}_{2} \mathrm{TIBi}$ are re-scaled by a factor of two for the purpose of visualization. The Fermi level in the band structures is shifted to zero octahedrally coordinated with six $\mathrm{Bi}(\mathrm{In} / \mathrm{Tl})$ as the next nearest neighbor. This is because $\mathrm{Li}$ is the most electropositive element in these compounds and it donates its $2 s$ electron to the crystal system. As shown in Fig. 2, Li $2 s$ electron is completely delocalized in these compounds and therefore it has very limited influence on the electronic structures of $\mathrm{Li}_{2} Y \mathrm{Bi}(Y=\mathrm{In}$ and $\mathrm{Tl})$ except for raising the Fermi level and opening the band gap, which is similar to the Li stabilized quaternary Heusler semiconductors $^{16}$. We further verified this conclusion by performing band structure calculations for $[\mathrm{TlBi}]^{2-}$ with the -2 charge balanced by a +2 Jellium background, see Supplementary Fig. 2 . Since $\mathrm{Tl}$ is the nearest neighbor of $\mathrm{Pb}$ and $\mathrm{Bi}$ is close to $\mathrm{Te}$ in the periodic table, $\mathrm{Li}_{2} \mathrm{InBi}$ and $\mathrm{Li}_{2} \mathrm{TlBi}$ have very similar band structures with $\mathrm{PbTe}$, as shown in Fig. $2 \mathrm{a}$. Interestingly, $\mathrm{Li}_{2} \mathrm{InBi}$ and $\mathrm{Li}_{2} \mathrm{TlBi}$ have much larger lattice constants than $\mathrm{PbTe}$ because of the inserted $\mathrm{Li}_{2}$ cubic sublattice, which plays an important role in raising the energy level of the VBM at the middle of $\Sigma$ line. As a consequence, the $N_{\mathrm{v}}$ of $\mathrm{Li}_{2} Y \mathrm{Bi}$ reaches to 12 , as observed in the $\mathrm{PbTe}$ under significant hydrostatic expansion. As depicted in Fig. 3, a remarkable decrease in the energy difference between $\Sigma$ and $\mathrm{L}$ is observed when the lattice constant of PbTe is expanded to that of $\mathrm{Li}_{2} \mathrm{TlBi}\left(\mathrm{Li}_{2} \mathrm{InBi}\right)$. At the same time, the large bonding distance (softer bonding interaction) between $\mathrm{Bi}$ and $Y(Y=\mathrm{In}$ and $\mathrm{Tl}$ ) contributes to reducing $\kappa_{\mathrm{L}}$ as we will see later ${ }^{5}$.

Since $\mathrm{Li}_{2} \mathrm{InBi}$ has a very similar electronic structure to $\mathrm{Li}_{2} \mathrm{TlBi}$, we only take $\mathrm{Li}_{2} \mathrm{TlBi}$ as an example here. The electronic structure of $\mathrm{Li}_{2} \mathrm{TlBi}$ is shown in Fig. 2 (the band structure of $\mathrm{Li}_{2} \mathrm{InBi}$ is shown in Supplementary Fig. 3 ). $\mathrm{Li}_{2} \mathrm{TlBi}$ is a small band gap semiconductor (PBE: $0.06 \mathrm{eV}$; HSE06: $0.18 \mathrm{eV}$, including the spin-orbit coupling (SOC), which is consistent with a previous calculation $^{25}$. These calculated gaps are well comparable with many high $z T$ TE materials, such as PbTe: $0.19 \mathrm{eV}^{26}$ and $\mathrm{CoSb}_{3}$ : $\left.0.05 \sim 0.22 \mathrm{eV}^{27,28}\right)$. In $\mathrm{Li}_{2} \mathrm{TlBi}$, the band gap opens between the fully occupied Bi $6 p$ and fully unoccupied $\mathrm{Tl} 6 p$ states due to charge transfer from $\mathrm{Tl}$ to $\mathrm{Bi}$. $\mathrm{Tl}$ atom loses its one $6 p$ electron to the more electronegative $\mathrm{Bi}$ atom and becomes $\mathrm{Tl}^{+}$, and its $6 s^{2}$ electrons are deeply ( $-5 \mathrm{eV}$ below the Fermi level) buried below the $\mathrm{Bi} 6 p$ orbitals (valence bands, from -4 to $0 \mathrm{eV}$ ), forming stereochemically inactive lone-pair electrons. Two electropositive $\mathrm{Li}$ atoms lose their $2 s$ electrons to $\mathrm{Bi}$ as well. Therefore, the $6 p$ orbitals of $\mathrm{Bi}^{3-}$ (from -4 to $0 \mathrm{eV}$ below the Fermi level) are fully filled with six electrons. The splitting of three occupied $\mathrm{Bi}$ $6 p$ orbitals into two groups, $\sim-2 \mathrm{eV}$ (single degeneracy) and $\sim-0.5 \mathrm{eV}$ (double degeneracy) at the $\Gamma$ point is due to SOC.
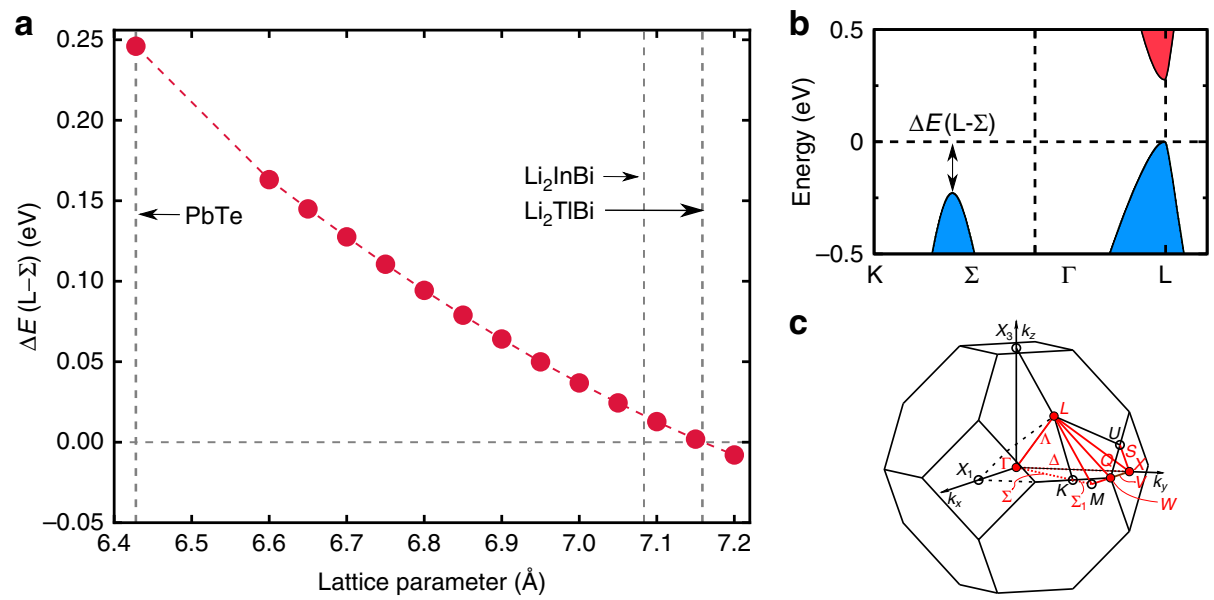

C

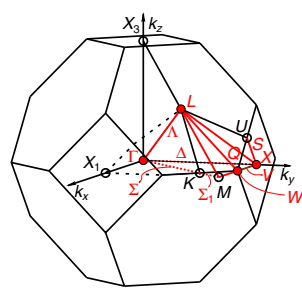

Fig. 3 Lattice induced band convergence. a The valence band energy difference between $\mathrm{L}$ and $\Sigma$ of PbTe as a function of lattice constant. The vertical lines indicates the lattice constants of experimental $\mathrm{PbTe}$ and fully relaxed $\mathrm{Li}_{2} \mathrm{TIBi}$ and $\mathrm{Li} 2 \mathrm{lnBi}$. $\mathbf{b}$ Band structure (blue: valence band; red: conduction band) of $\mathrm{PbTe}$ along K- $\Gamma-\mathrm{L}$ direction. $\mathbf{c}$ High symmetry points/line of the first Brillouin zone of $F m \overline{3} m$ 


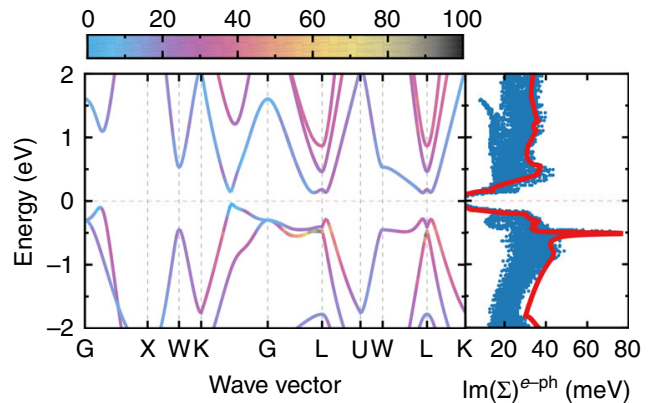

Fig. 4 Electron-phonon coupling. Heat map of the imaginary part of the electron-phonon self-energy $(\operatorname{Im}(\Sigma))$ of $\mathrm{Li}_{2} \mathrm{TIBi}$ at $300 \mathrm{~K}$ (left) and modedependent $\operatorname{Im}(\Sigma)$ compared with scaled density of states (DOS) (right). Fermi level is shifted to zero

The conduction bands are mainly from the $\mathrm{Tl}^{+} 6 p$ orbitals. The electron localization function (ELF) is shown in Fig. 1 and Supplementary Fig. 4. We can clearly see that $\mathrm{Bi}$ and $\mathrm{Li}$ atoms have, respectively, the highest and lowest ELF values, consistent with our electronic structure analysis that Li donates its electrons to the system while $\mathrm{Bi}$ gains electrons. The inactive lone-pair electrons of $\mathrm{Tl}^{+} 6 s^{2}$ are clearly observed in Fig. 1 as well, which is analogous to $\mathrm{Pb}^{2+}$ in PbTe. Finally, the ELF of $\mathrm{Li}_{2} \mathrm{TlBi}$ is very similar to that of $\mathrm{PbTe}$, which is identified as a typical resonant bonding system ${ }^{29,30}$.

As expected from the previous analysis, a remarkable feature of the $\mathrm{Li}_{2} \mathrm{TlBi}$ band structure is that its VBM lies in the middle of $\Sigma$ line of the first Brillouin zone of the FCC FH structure $(F m \overline{3} m)$, which leads to an unexpected high valley degeneracy $\left(N_{\mathrm{v}}=12\right)$, see the Fermi surface in Fig. 2. Hence the $N_{\mathrm{v}}=12$ of the VBM reaches a record high value, which only has been previously matched in the heavily doped $\mathrm{PbTe}$ and $\mathrm{CoSb}_{3}$ systems ${ }^{8,31}$. The second hole pocket, which is $\sim 40 \mathrm{meV}$ lower than VBM, is located at the middle of the $\Delta$ line (between $\Gamma$ and $\mathrm{X}$ ) and possesses a valley degeneracy of 6 . Therefore, an extremely high $N_{\mathrm{v}}=18$ is reachable in $\mathrm{Li}_{2} \mathrm{TlBi}$ by means of hole doping. Although the CBM is located at $\mathrm{L}$ with the valley degeneracy of 4 , the energy difference between CBM and the second highest electron pocket (in the middle of the $\Sigma$ line) is only $7 \mathrm{meV}$. Therefore the $N_{\mathrm{v}}$ of the conduction band can potentially reach as high as 16 through light electron doping. The Fermi surfaces of the valence and conduction bands are displayed in Fig. 2. As mentioned above, although the band effective masses $\left(m_{\mathrm{b}}^{*}\right)$ for the VBM and CBM are small, which imply high carrier mobilities as $\mu \propto \frac{\tau}{m^{*}}$, the Seebeck coefficient $S \propto m_{\mathrm{d}}^{*}$ still can be very high, provided $^{m_{\mathrm{b}}} N_{\mathrm{v}}$ is sufficiently large, since $m_{\mathrm{d}}^{*}$ is related to the band effective mass by $m_{\mathrm{d}}^{*}=N_{\mathrm{v}}^{2 / 3} m_{\mathrm{b}}^{*}$.

Electron transport. To quantitatively characterize the electron transport properties of $\mathrm{Li}_{2} Y \mathrm{Bi}(Y=\mathrm{In}$ and $\mathrm{Tl})$, we calculate $S$ and $\sigma$ based on the semiclassical Boltzmann transport equation under relaxation time approximation. We assume that the predominant carrier scattering mechanisms at $200 \mathrm{~K}$ and above are all based on phonons: (1) deformation potentials of acoustic and optical phonons and (2) Fröhlich coupling due to polar optical phonons ${ }^{32,33}$. Since the best thermoelectric efficiency is always achieved in the heavily doped region where the scattering on polar optical phonons is sufficiently screened and the dielectric constant is usually large in narrow band gap semiconductors ${ }^{32-34}$, we mainly take into account deformation potential scattering using first-principles calculated electron-phonon interaction (EPI) matrix elements. As shown in Fig. 4 for the representative compound $\mathrm{Li}_{2} \mathrm{TlBi}$, the imaginary part of the electron self-energy $\operatorname{Im}(\Sigma)$ shows a strong energy dependence and is roughly proportional to the density of electronic states. States with a long lifetime appear near the VBM and CBM. This indicates that the lifetime is linked to the phase space availability for electronic transitions, i.e., electrons and holes near the band edges are less scattered due to limited phase space ${ }^{35}$.

To validate our calculations, we also computed the thermoelectric properties for a well studied p-type $\mathrm{HH}$ compound FeNbSb, for which a PF as large as $10.6 \mathrm{~mW} \mathrm{~m}^{-1} \mathrm{~K}^{-2}$ was recently measured at room temperature ${ }^{36}$. Figure $5 \mathrm{c}$ shows that our calculation considering electron-phonon coupling predicts a maximum $\mathrm{PF}$ of $11.7 \mathrm{~mW} \mathrm{~m}^{-1} \mathrm{~K}^{-2}$ for $\mathrm{FeNbSb}$ at $300 \mathrm{~K}$, representing the upper limit without considering other scattering sources such as defects and grain boundaries. Our calculated $S, \sigma$, and $\mathrm{PF}$ of $\mathrm{FeNbSb}$ at optimized carrier concentration and temperatures from 200 to $500 \mathrm{~K}$ also compare well with a recent theoretical study that employs the same methodology ${ }^{37}$. The good agreement between our calculations and experimental data confirms our assumption that electron-phonon coupling dominates carrier scattering in this system. It is noteworthy that the optimal PF of FeNbSb is significantly higher than that of PbTe at $300 \mathrm{~K}^{8,38,39}$.

Next, we illustrate the ultrahigh PFs of $\mathrm{Li}_{2} \mathrm{TlBi}$ and $\mathrm{Li}_{2} \mathrm{InBi}$ by comparing to FeNbSb. Despite the fact that $S$ is generally much higher in FeNbSb (see Supplementary Figs. 5 and 6) due to its larger band gap of $0.54 \mathrm{eV}$ compared to $0.18 \mathrm{eV}\left(\mathrm{Li}_{2} \mathrm{TlBi}\right)$ and $0.15 \mathrm{eV}\left(\mathrm{Li}_{2} \mathrm{InBi}\right)$, the $S$ of $\mathrm{Li}_{2} \mathrm{TlBi}$ and $\mathrm{Li}_{2} \mathrm{InBi}$ is comparable with that of FeNbSb at optimal carrier concentration, particularly at $300 \mathrm{~K}$, as shown in Fig. 5a. The strong bipolar effect further suppresses $S$ of $\mathrm{Li}_{2} \mathrm{TlBi}$ and $\mathrm{Li}_{2} \mathrm{InBi}$ at higher temperatures. However, owing to the smaller band effective mass $\left(m_{\mathrm{b}}^{*}\right)$ and high valley degeneracy $\left(N_{\mathrm{v}}\right)$, both $\mathrm{Li}_{2} \mathrm{TlBi}$ and $\mathrm{Li}_{2} \mathrm{InBi}$ have significantly higher $\sigma$ than FeNbSb from 300 to $500 \mathrm{~K}$ with a carrier concentration about one order of magnitudue lower than FeNbSb (see Supplementary Figs. 5 and 6). As a consequence, $\mathrm{Li}_{2} \mathrm{TlBi}\left(\mathrm{Li}_{2} \mathrm{InBi}\right)$ achieves exceptional PFs of $30.4 / 20.1(26.3 / 19.0) \mathrm{mW} \mathrm{m}^{-1} \mathrm{~K}^{-2}$ at $200 / 300 \mathrm{~K}$, nearly twice that of $\mathrm{FeNbSb}$ at $300 \mathrm{~K}$. The outperformance of $\mathrm{Li}_{2} \mathrm{TlBi}$ and $\mathrm{Li}_{2} \mathrm{InBi}$ over FeNbSb is due to a comparable $S$ and a higher $\sigma$ at the optimized carrier concentrations, supporting our previous discussion.

Phonon transport. The $\mathrm{Li}_{2} \mathrm{TlBi}\left(\mathrm{Li}_{2} \mathrm{InBi}\right)$ primitive cell contains four atoms and therefore 12 phonon branches. The mode decomposition in the zone center ( $\Gamma$ point) is $3 \mathrm{~T}_{1 u} \oplus 1 \mathrm{~T}_{2 g}$. As shown in Fig. 6, the low-frequency phonon modes are mainly from the stereochemically inert lone-pair $\mathrm{Tl}^{+}$cation instead of the heaviest atom $\mathrm{Bi}$, which is consistent with the weaker bonding between $\mathrm{Tl}$ atom and its neighbors. As expected, the light lithium atom has much higher phonon frequencies $200 \sim 250 \mathrm{~cm}^{-1}$ and its phonon bands are completely separated from $\mathrm{Tl}$ and $\mathrm{Bi}$. It is worth noting that these compounds possess two main differences from the previously reported alkali metal based rattling $(R)$ Heusler ${ }^{15}$ : (i) higher acoustic phonon frequencies, and (ii) higher frequency of crossing bands between acoustic and optical modes, meaning $\mathrm{Tl}$ (In) atom has a slightly stronger interaction with its neighbors than $R$-Heusler compounds.

The lattice thermal conductivity $\kappa_{\mathrm{L}}$ is calculated by using firstprinciples compressive sensing lattice dynamics (CSLD) and solving the linear Boltzmann transport equation (see Methods for details) and the results are shown in Fig. 7. Owing to the cubic symmetry, $\kappa_{\mathrm{L}}$ of $\mathrm{Li}_{2} \mathrm{TlBi}$ and $\mathrm{Li}_{2} \mathrm{InBi}$ are isotropic $\left(\kappa_{\mathrm{L}}^{x x}=\kappa_{\mathrm{L}}^{y y}=\kappa_{\mathrm{L}}^{z z}\right.$ $\left.=\kappa_{\mathrm{L}}\right)$ and the calculated $\kappa_{\mathrm{L}}$ are $2.36(1.55) \mathrm{Wm}^{-1} \mathrm{~K}^{-1}$ at $300 \mathrm{~K}$ and $0.55(0.52) \mathrm{Wm}^{-1} \mathrm{~K}^{-1}$ at $900 \mathrm{~K}$ for $\mathrm{Li}_{2} \mathrm{TlBi}\left(\mathrm{Li}_{2} \mathrm{InBi}\right)$, which 

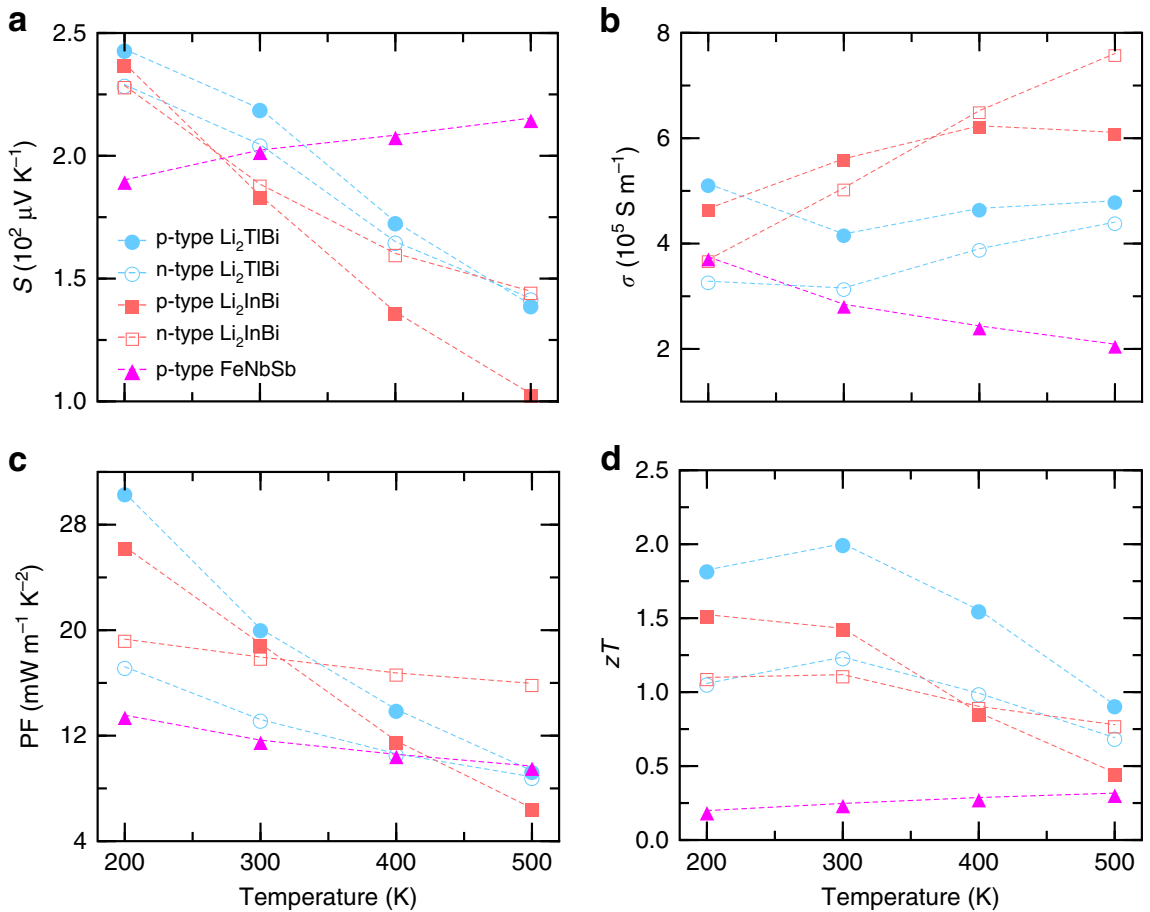

Fig. 5 Electron transport properties. a-d The Seebeck coefficient $S(\mathbf{a})$, electrical conductivity $\sigma(\mathbf{b})$, power factor PF (c), and $z T$ (d) of Li ${ }_{2} T I B i$ and Li 2 InBi at carrier concentrations that give rise to maximum $z T$ at 200,300,400, and $500 \mathrm{~K}$, compared with p-type FeNbSb. The lattice thermal conductivity of FeNbSb used to compute $z T$ is extracted from ref. ${ }^{36}$. The electronic thermal conductivity $\left(\kappa_{\mathrm{e}}\right)$ is shown in Supplementary Figs. 4 and 5

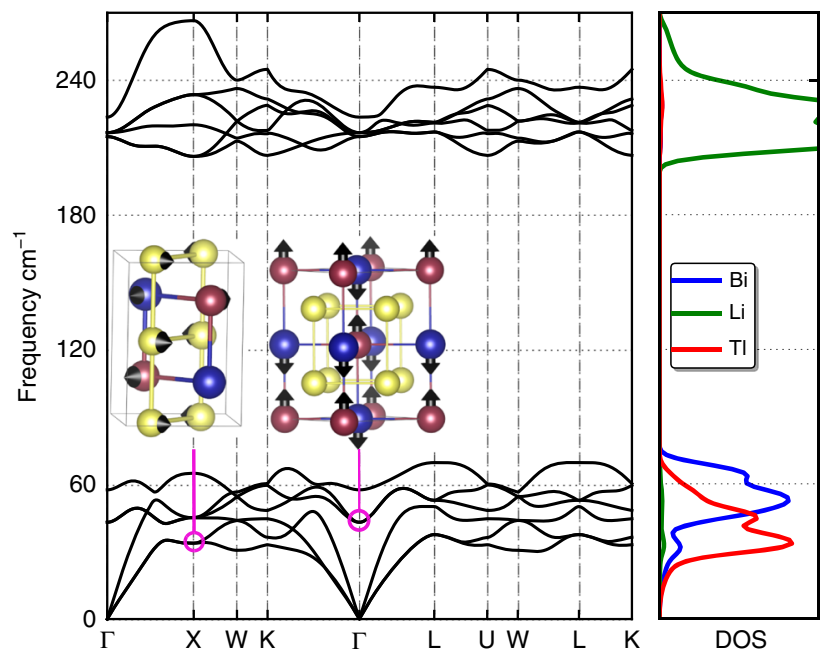

Fig. 6 Phonon dispersion. Phonon spectra (left) and phonon density of states (right) of $\mathrm{Li}_{2} \mathrm{TIBi}$. The longitudinal optical (LO) and transverse optical (TO) splitting is included. Insets are the phonon vibration models in real space at the $k$-point and energy indicated by pink circle

are much lower than most $\mathrm{FH}$ and $\mathrm{HH}\left(\geq 7 \mathrm{Wm}^{-1} \mathrm{~K}^{-140}\right)$ compounds without doping or nanostructuring and also lower than PbTe $\left(2.74\right.$ at $300 \mathrm{~K}$ and $0.91 \mathrm{Wm}^{-1} \mathrm{~K}^{-1}$ at $900 \mathrm{~K}$ at the same computational level).

Similar to $\mathrm{PbTe}, \mathrm{Li}_{2} \mathrm{InBi}$, and $\mathrm{Li}_{2} \mathrm{TlBi}$ have low-lying transverse optical modes (TO), as shown in Supplementary Figs. 7 and 8, further confirming the presence of the resonant bonding ${ }^{29}$, as expected from earlier electronic structures analysis. The longrange interaction caused by the resonant bonding leads to strong anharmonic scattering and large phase space for three-phonon scattering processes and, therefore, significantly suppresses lattice thermal transport ${ }^{29}$. Moreover, the weak Tl-Bi (In-Bi) bonding resulting from the large bonding distance between $\mathrm{Tl}$ and $\mathrm{Bi}$ (In and $\mathrm{Bi}$ ) gives rise to low group velocities. Finally, the high-frequency optical modes associated with the $\mathrm{Li}$ atoms provide extra scattering channels for low-lying acoustic modes.

The mechanism of the strong scattering of heat carrying acoustic phonon modes can be directly understood from phonon-phonon interactions. We show the phonon-phonon scattering rates in the absorption $\left(\Gamma^{+}: \lambda+\lambda^{\prime} \rightarrow \lambda^{\prime \prime}\right)$ and emission $\left(\Gamma^{-}: \lambda^{\prime \prime} \rightarrow \lambda+\lambda^{\prime}\right)$ processes in Fig. 7. The low-frequency acoustic phonons are mainly scattered by the low-frequency optical modes in the absorption process, while the optical modes decompose largely into low energy acoustic modes in the emission process. This scattering picture is similar to the alkali metal based $R$-Heusler compounds ${ }^{15}$.

\section{Discussion}

Using our calculated $\kappa_{\mathrm{L}}, S, \sigma$, and $\kappa_{\mathrm{e}}$ within DFT framework by explicitly including electron-phonon and phonon-phonon interactions, the maximum figure of merit $z T$ of $\mathrm{Li}_{2} \mathrm{TlBi}$ and $\mathrm{Li}_{2} \mathrm{InBi}$ are estimated to be 2.0 and 1.4 at $300 \mathrm{~K}$ for hole doping (p-type), respectively, which implies that $\mathrm{Li}_{2} \mathrm{TlBi}$ is the TE material with the highest $z T$ at room temperature. Note that our calculated $\kappa_{\mathrm{L}}$ might be overestimated due to neglect of the phonon-phonon interaction beyond the third-order and phonon scattering by defects and grain boundaries. Furthermore, the $z T$ of these FH materials could be further enhanced by suppressing heat transport through nanostructuring precipitates and optimizing grain size as commonly used in other Heusler compounds if the optimized carrier concentration can still be achieved. The optimized hole concentrations within the rigid band approximation for the maximum $z T$ at room temperature are $1.3 \times 10^{19}$ and $1.6 \times 10^{19} \mathrm{~cm}^{-3}$, respectively, for $\mathrm{Li}_{2} \mathrm{TlBi}$ and $\mathrm{Li}_{2} \mathrm{InBi}$ (see Supplementary Figs. 5 and 6), which is close to those for PbTe at room temperature 38,39 but one order of magnitude lower than in 

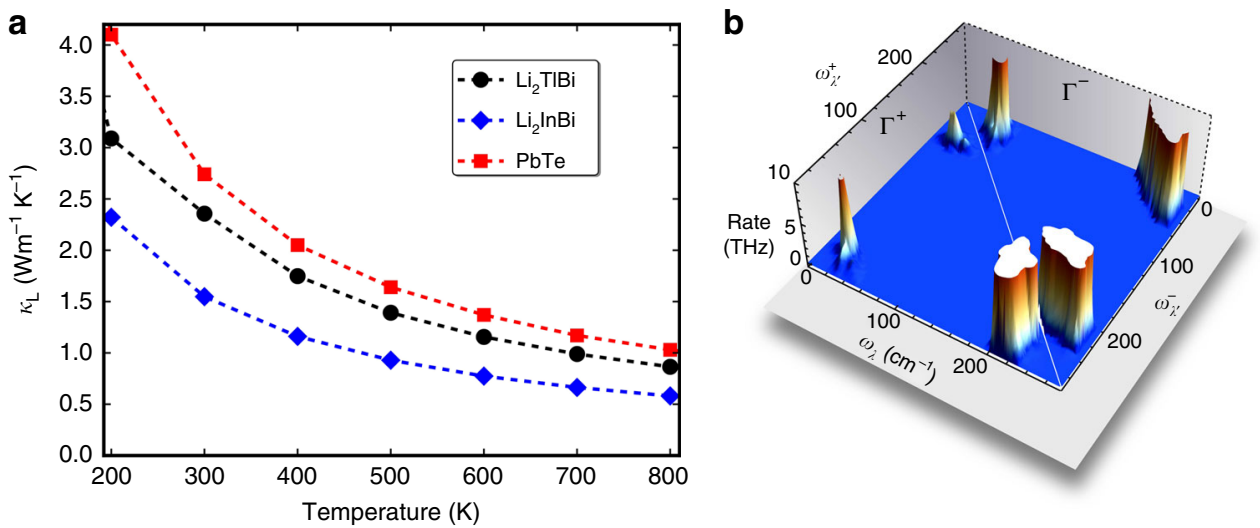

Fig. 7 Phonon transport properties. a Calculated lattice thermal conductivity $\kappa_{\mathrm{L}}$ of $\mathrm{Li}_{2} \mathrm{TIBi}, \mathrm{Li}_{2} \mathrm{InBi}$, and $\mathrm{PbTe}$ as function of temperature. b Phonon scattering rates of $\mathrm{Li}_{2} \mathrm{TIBi}$ in absorption $\left(\Gamma^{+}: \lambda+\lambda^{\prime} \rightarrow \lambda^{\prime \prime}\right)$ and emission $\left(\Gamma^{-}: \lambda^{\prime \prime} \rightarrow \lambda+\lambda^{\prime}\right)$ processes

$\mathrm{FeNbSb}^{36}$. Since $\mathrm{Li}_{2} \mathrm{TlBi}$ has a better electronic structure than $\mathrm{PbTe}$, the maximum $z T$ at the same carrier concentration and room temperature is also higher ${ }^{39}$.

For experiments, it is important to ascertain thermodynamic limit to the achievable dopant concentration. To estimate the defect solubility of forming p-type semiconductor, neutral defects of $\mathrm{Li}$ and $\mathrm{Tl}$ vacancy were considered. The calculated vacancy formation energy $\left(E_{\mathrm{d}}\right)$ of $\mathrm{Li}(\mathrm{Tl})$ in $\mathrm{Li}_{2} \mathrm{TlBi}$ is $0.29(0.31) \mathrm{eV}$ per vacancy in $\mathrm{Li}(\mathrm{Tl})$ poor condition. These values are comparable with that of the $\mathrm{Na}$ doped $\mathrm{PbTe}(0.27 \mathrm{eV}$ per defect) 41 , where the hole concentration can reach $10^{20} \mathrm{~cm}^{-3}$ at room temperature ${ }^{42,43}$. These values strongly suggest that the required hole concentrations for maximizing $z T$ are achievable in $\mathrm{Li}_{2} \mathrm{TlBi}$.

Owing to the small band gap, the maximum $z T$ values of $\mathrm{Li}_{2} \mathrm{TlBi}$ and $\mathrm{Li}_{2} \mathrm{InBi}$ are at room temperature, see Fig. 5. The drop down of the $z T$ at higher temperature is mainly due to the decreased PF by the bipolar effect, stemming from their small band gaps since we assume that the band gap does not change with temperature. If their band gaps widen with elevated temperature as observed in $\mathrm{PbTe}$, the maximum $z T$ will be achieved at higher temperature. We also note that the electron doped (n-type) $\mathrm{Li}_{2} \mathrm{TlBi}$ and $\mathrm{Li}_{2} \mathrm{InBi}$ have high $z T$ at room temperature as well, due to the high conduction band degeneracy (at $\Sigma$ line and $\mathrm{L}$ point) and low lattice thermal conductivity. A material with high $z T$ for both hole and electron doping is important for fabricating TE devices. Therefore, $\mathrm{Li}_{2} \mathrm{TlBi}$ and $\mathrm{Li}_{2} \mathrm{InBi}$ are promising materials for room temperature thermoelectric applications.

In summary, we discover two promising room-temperature TE materials, $\mathrm{Li}_{2} \mathrm{TlBi}$ and $\mathrm{Li}_{2} \mathrm{InBi} \mathrm{FHs}$, by high throughput stability screening and TE material design strategy of creating the analogs with isovalent electronic structures to $\mathrm{PbTe}$ with much expanded lattices. We demonstrate that $\mathrm{Li}_{2} \mathrm{TlBi}$ and $\mathrm{Li}_{2} \mathrm{InBi}$ possess intrinsic high PFs and low $\kappa_{\mathrm{L}}$ by using the state-of-theart computational methods which combines the electron Boltzmann transport theory with ab initio carrier relaxation-time from electron-phonon coupling and phonon transport theory with phonon lifetime from first-principles CSLD. The high TE performance of the p-type $\mathrm{Li}_{2} \mathrm{TlBi}$ and $\mathrm{Li}_{2} \mathrm{InBi}$ at room temperature are mainly due to the high $N_{\mathrm{v}}$ induced by the extended lattice and the low $\kappa_{\mathrm{L}}$ caused by the resonant bonding as observed in $\mathrm{PbTe}$ and weak bonding interactions of the extended lattice, respectively. Our TE material design strategy enhances the band degeneracy and suppresses lattice thermal conductivity of the $\mathrm{PbTe}$-type materials simultaneously. It is also straightforward to be extended to design or discover other TE materials.

\section{Methods}

DFT calculation details. In this study, most DFT calculations are performed using the Vienna Ab initio Simulation Package (VASP) ${ }^{44,45}$. The projector augmented wave $\left(\mathrm{PAW}^{46,47}\right)$ pseudo potential, plane wave basis set, and

Perdew-Burke-Ernzerhof $\left(\mathrm{PBE}^{48}\right)$ exchange-correlation functional were used. The $\mathrm{qmpy}^{18}$ framework and the Open Quantum Material Database (OQMD) ${ }^{18}$ was used for convex hull construction. The band gap was computed by means of the screened hybrid functional HSE06 ${ }^{49}$, including spin-orbit coupling (SOC).

Crystal structure prediction. The lowest energy structure of $\mathrm{Li}_{2} Y \mathrm{Bi}$ were confirmed by prototype structure screening ${ }^{15}$ and crystal structure searching using the particle swarm optimization method as implemented in the CALYPSO code 21,22 .

Free energy calculations. The lattice dynamic stability and vibrational entropy were computed by performing frozen phonon calculation as implemented in phonopy package ${ }^{50}$. The disordered competing phases were simulated using the special quasirandom structures (SQS) as implemented in the alloy theoretic automated toolkit (ATAT) ${ }^{51}$. Vibrational entropies of the ordered phases and SQSs of the disordered phases are calculated by using phonopy package ${ }^{50}$. Configuration entropy is included if the competing phases have atom disorder. The calculated results are shown in the Supplementary Table 2.

Phonon and electron transport calculations. The $\operatorname{CSLD}^{52}$ technique was employed to obtain the third-order force constants, which were used to iteratively solve the linearized phonon Boltzmann transport equation with the ShengBTE package $^{53}$. The carrier lifetime due to electron-phonon coupling was computed by using Quantum Espresso and Electron-phonon Wannier (EPW) codes with SOC included ${ }^{35,54-56}$. Thermoelectric properties including Seebeck coefficient $(S)$, electrical conductivity $(\sigma)$, and electronic thermal conductivity $\left(\kappa_{\mathrm{e}}\right)$ were computed using BoltzTrap code ${ }^{57}$ with the adjusted band gap from HSE06 calculations and mode-resolved carrier lifetime from EPW.

\section{Data availability}

All data are available from the corresponding authors upon reasonable request. All codes used in this work are either publicly available or available from the authors upon reasonable request.

Received: 15 May 2018 Accepted: 3 January 2019

Published online: 12 February 2019

\section{References}

1. Toberer, E. S., Zevalkink, A. \& Snyder, G. J. Phonon engineering through crystal chemistry. J. Mater. Chem. 21, 15843-15852 (2011).

2. Pei, Y., Wang, H. \& Snyder, G. Band engineering of thermoelectric materials. Adv. Mater. 24, 6125-6135 (2012)

3. He, J. et al. $\mathrm{Bi}_{2} \mathrm{PdO}_{4}$ : a promising thermoelectric oxide with high power factor and low lattice thermal conductivity. Chem. Mater. 29, 2529-2534 (2017).

4. Usui, H. \& Kuroki, K. Enhanced power factor and reduced lorenz number in the wiedemann-franz law due to pudding mold type band structures. J. Appl. Phys. 121, 165101 (2017). 
5. Zeier, W. G. et al. Thinking like a chemist: intuition in thermoelectric materials. Angew. Chem. Int. Ed. Engl. 55, 6826-6841 (2016).

6. Snyder, G. J. \& Toberer, E. S. Complex thermoelectric materials. Nat. Mater. 7, 105-114 (2008).

7. Gogotsi, Y. Nanomaterials Handbook. (CRC Press, Boca Raton, 2006).

8. Pei, Y. et al. Convergence of electronic bands for high performance bulk thermoelectrics. Nature 473, 66-69 (2011).

9. Zhu, H., Sun, W., Armiento, R., Lazic, P. \& Ceder, G. Band structure engineering through orbital interaction for enhanced thermoelectric power factor. Appl. Phys. Lett. 104, 082107 (2014).

10. Sootsman, J. R., Chung, D. Y. \& Kanatzidis, M. G. New and old concepts in thermoelectric materials. Angew. Chem. Int. Ed. Engl. 48, 8616-8639 (2009).

11. Yang, J. et al. Evaluation of half-Heusler compounds as thermoelectric materials based on the calculated electrical transport properties. Adv. Funct. Mater. 18, 2880-2888 (2008).

12. Fu, C. et al. High band degeneracy contributes to high thermoelectric performance in p-type half-heusler compounds. Adv. Energy Mater. 4, 1400600 (2014).

13. Fu, C. et al. Realizing high figure of merit in heavy-band p-type half-heusler thermoelectric materials. Nat. Commun. 6, 8144 (2015).

14. Fu, C., Zhu, T., Liu, Y., Xie, H. \& Zhao, X. Band engineering of high performance p-type fenbsb based half-heusler thermoelectric materials for figure of merit zt > 1. Energy Environ. Sci. 8, 216-220 (2015).

15. He, J. et al. Ultralow thermal conductivity in full heusler semiconductors. Phys. Rev. Lett. 117, 046602 (2016).

16. He, J., Naghavi, S. S., Hegde, V. I., Amsler, M. \& Wolverton, C. Designing and discovering a new family of semiconducting quaternary heusler compounds based on the 18-electron rule. Chem. Mater. 30, 4978-4985 (2018) https:// pubs.acs.org/doi/abs/10.1021/acs.chemmater.8b01096.

17. Kirklin, S., Saal, J. E., Hegde, V. I. \& Wolverton, C. High-throughput computational search for strengthening precipitates in alloys. Acta Mater. 102, 125-135 (2016).

18. Saal, J., Kirklin, S., Aykol, M., Meredig, B. \& Wolverton, C. Materials design and discovery with high-throughput density functional theory: the open quantum materials database (oqmd). JOM 65, 1501-1509 (2013).

19. Bergerhoff, G. \& Brown, I. D. in Crystallographic databases. 77-95 (International Union of Crystallography, Chester, 1987).

20. Belsky, A., Hellenbrandt, M., Karen, V. L. \& Luksch, P. New developments in the Inorganic Crystal Structure Database (ICSD): accessibility in support of materials research and design. Acta Cryst. B58, 364-369 (2002).

21. Wang, Y., Lv, J., Zhu, L. \& Ma, Y. Crystal structure prediction via particleswarm optimization. Phys. Rev. B 82, 094116 (2010).

22. Wang, Y., Lv, J., Zhu, L. \& Ma, Y. Calypso: a method for crystal structure prediction. Comput. Phys. Commun. 183, 2063-2070 (2012).

23. $\mathrm{Ma}$, J. et al. Computational investigation of half-heusler compounds for spintronics applications. Phys. Rev. B 95, 024411 (2017).

24. Ma, J. et al. Computational investigation of inverse heusler compounds for spintronics applications. Phys. Rev. B 98, 094410 (2018).

25. Pham, A. \& Li, S. Unique topological surface states of full-heusler topological crystalline insulators. Phys. Rev. B 95, 115124 (2017).

26. Skelton, J. M., Parker, S. C., Togo, A., Tanaka, I. \& Walsh, A. Thermal physics of the lead chalcogenides $\mathrm{PbS}, \mathrm{PbSe}$, and PbTe from first principles. Phys. Rev. B 89, 205203 (2014).

27. Singh, D. J. \& Pickett, W. E. Skutterudite antimonides: quasilinear bands and unusual transport. Phys. Rev. B 50, 11235-11238 (1994).

28. Sofo, J. O. \& Mahan, G. D. Electronic structure of $\mathrm{CoSb}_{3}$ : a narrow-band-gap semiconductor. Phys. Rev. B 58, 15620-15623 (1998).

29. Lee, S. et al. Resonant bonding leads to low lattice thermal conductivity. Nat. Commun. 5, 3525 (2014).

30. Shportko, K. et al. Resonant bonding in crystalline phase-change materials. Nat. Mater. 7, 653 (2008).

31. Tang, Y. et al. Convergence of multi-valley bands as the electronic origin of high thermoelectric performance in cosb3 skutterudites. Nat. Mater. 14, 1223-1229 (2015).

32. Freik, D. M., S, V. \& Nykyruy, L. I. Scattering mechanisms of electrons in monocrystalline PbTe, PbSe and PbS. Semicond. Phys. Quantum Electron. Optoelectron. 5, 362 (2002).

33. Vineis, C. J. et al. Carrier concentration and temperature dependence of the electronic transport properties of epitaxial $\mathrm{PbTe}$ and $\mathrm{PbTe} / \mathrm{PbSe}$ nanodot superlattices. Phys. Rev. B 77, 235202 (2008).

34. Leite Alves, H. W., Neto, A. R. R., Scolfaro, L. M. R., Myers, T. H. \& Borges, P. D. Lattice contribution to the high dielectric constant of PbTe. Phys. Rev. B 87, 115204 (2013)

35. Giustino, F., Cohen, M. L. \& Louie, S. G. Electron-phonon interaction using wannier functions. Phys. Rev. B 76, 165108 (2007).
36. He, R. et al. Achieving high power factor and output power density in p-type half-heuslers $\mathrm{Nb}_{1-x} \mathrm{Ti}_{x}$ FeSb. Proc. Natl Acad. Sci. USA 113, 13576-13581 (2016).

37. Zhou, J. et al. Large thermoelectric power factor from crystal symmetryprotected non-bonding orbital in half-heuslers. Nat. Commun. 9, 1721 (2018).

38. Pei, Y. et al. Optimum carrier concentration in n-type PbTe thermoelectrics. Adv. Energy Mater. 4, 1400486 (2014).

39. Pei, Y., Wang, H., Gibbs, Z. M., LaLonde, A. D. \& Snyder, G. J. Thermopower enhancement in $\mathrm{Pb}_{1-x} \mathrm{Mn}_{x}$ Te alloys and its effect on thermoelectric efficiency. NPG Asia Mater. 4, e28 (2012).

40. Chen, S. \& Ren, Z. Recent progress of half-heusler for moderate temperature thermoelectric applications. Mater. Today 16, 387-395 (2013).

41. He, J. et al. Role of sodium doping in lead chalcogenide thermoelectrics. J. Am. Chem. Soc. 135, 4624-4627 (2013).

42. Wang, H., Pei, Y., LaLonde, A. D. \& Snyder, G. J. Heavily doped p-type PbSe with high thermoelectric performance: An alternative for PbTe. Adv. Mater. 23, 1366-1370 (2011).

43. Wang, X. et al. Sodium substitution in lead telluride. Chem. Mater. 30, 1362-1372 (2018).

44. Kresse, G. \& Hafner, J. Ab initio molecular dynamics for open-shell transition metals. Phys. Rev. B 48, 13115-13118 (1993).

45. Kresse, G. \& Furthmülerb, J. Efficiency of ab-initio total energy calculations for metals and semiconductors using a plane-wave basis set. Comp. Mater. Sci. 6, 15 (1996).

46. Blöchl, P. E. Projector augmented-wave method. Phys. Rev. B 50, 17953-17979 (1994).

47. Kresse, G. \& Joubert, D. From ultrasoft pseudopotentials to the projector augmented-wave method. Phys. Rev. B 59, 1758-1775 (1999).

48. Perdew, J. P., Burke, K. \& Ernzerhof, M. Generalized gradient approximation made simple. Phys. Rev. Lett. 77, 3865-3868 (1996).

49. Heyd, J., Scuseria, G. E. \& Ernzerhof, M. Hybrid functionals based on a screened coulomb potential. J. Chem. Phys. 118, 8207-8215 (2003); erratum 124, 219906 (2006).

50. Togo, A., Oba, F. \& Tanaka, I. First-principles calculations of the ferroelastic transition between rutile-type and $\mathrm{CaCl}_{2}$-type $\mathrm{SiO}_{2}$ at high pressures. Phys. Rev. B 78, 134106 (2008)

51. van de Walle, A. Multicomponent multisublattice alloys, nonconfigurational entropy and other additions to the alloy theoretic automated toolkit. Calphad 33, 266-278 (2009).

52. Zhou, F., Nielson, W., Xia, Y. \& Ozoliņš, V. Lattice anharmonicity and thermal conductivity from compressive sensing of first-principles calculations. Phys. Rev. Lett. 113, 185501 (2014).

53. Li, W., Carrete, J., Katcho, N. A. \& Mingo, N. ShengBTE: a solver of the Boltzmann transport equation for phonons. Comp. Phys. Commun. 185, 1747-1758 (2014).

54. Giannozzi, P. et al. Quantum espresso: a modular and open-source software project for quantum simulations of materials. J. Phys. Condens. Matter 21, 395502 (2009).

55. Poncé, S., Margine, E. R., Verdi, C. \& Giustino, F. EPW: electron-phonon coupling, transport and superconducting properties using maximally localized wannier functions. Comput. Phys. Commun. 209, 116-133 (2016).

56. Giustino, F. Electron-phonon interactions from first principles. Rev. Mod. Phys. 89, 015003 (2017).

57. Madsen, G. K. H. \& Singh, D. J. Boltztrap. a code for calculating bandstructure dependent quantities. Comput. Phys. Commun. 175, 67-71 (2006).

\section{Acknowledgements}

J.H. and C.W. (stabilities and electronic structures calculations) acknowledge support by the U.S. Department of Energy, Office of Science and Office of Basic Energy Sciences, under Award No. DE-SC0014520. Y.X. (lattice thermal conductivity and electron transport calculations), S.S.N. (electronic structure analysis), and V.O. (electronic structure analysis) were supported by US Department of Energy, Office of Science, Basic Energy Sciences, under grant DE-FG02-07ER46433. This research used resources of the National Energy Research Scientific Computing Center, a DOE Office of Science User Facility supported by the Office of Science of the U.S. Department of Energy under Contract No. DE-AC02-05CH11231.

\section{Author contributions}

The research was conceived and designed by J.H., V.O., and C.W. High throughput DFT screening, stabilities, and electronic structure calculations were carried out by J.H. Thermoelectric properties calculations were conducted by Y.X. Analysis of the data was performed by J.H., S.S.N., and Y.X. All authors discussed the results contributed to writing the manuscript. 


\section{Additional information}

Supplementary Information accompanies this paper at https://doi.org/10.1038/s41467019-08542-1.

Competing interests: The authors declare no competing interests.

Reprints and permission information is available online at http://npg.nature.com/ reprintsandpermissions/

Journal peer review information: Nature Communications thanks the anonymous reviewers for their contribution to the peer review of this work.

Publisher's note: Springer Nature remains neutral with regard to jurisdictional claims in published maps and institutional affiliations. (c) Open Access This article is licensed under a Creative Commons Attribution 4.0 International License, which permits use, sharing, adaptation, distribution and reproduction in any medium or format, as long as you give appropriate credit to the original author(s) and the source, provide a link to the Creative Commons license, and indicate if changes were made. The images or other third party material in this article are included in the article's Creative Commons license, unless indicated otherwise in a credit line to the material. If material is not included in the article's Creative Commons license and your intended use is not permitted by statutory regulation or exceeds the permitted use, you will need to obtain permission directly from the copyright holder. To view a copy of this license, visit http://creativecommons.org/ licenses/by/4.0/.

(C) The Author(s) 2019 